Can. J. Fish. Aquat. Sci

2006; 63(6) : 1414-1428

http://dx.doi.org/10.1139/Z

(c) 2005 NRC Canada
Archimer http://www.ifremer.fr/docelec/ Archive Institutionnelle de l'Ifremer

The original publication is available at http://pubs.nrc-cnrc.gc.ca

\title{
Semi-local extraction of ring structures in images of biological hard tissues: application to the Bayesian interpretation of fish otoliths for age and growth estimation
}

\section{Ronan Fablet}

Ifremer/LASAA, BP 70, 29280 Plouzané,France, France

*: Corresponding author : $\underline{\text { rfablet@ifremer.fr }}$

\begin{abstract}
This paper deals with the analysis of images of biological tissue that involves ring structures, such as tree trunks, bivalve seashells, or fish otoliths, with a view to automating the acquisition of age and growth data. A bottom-up template-based scheme extracts meaningful ridge and valley curve data using growth-adapted time-frequency filtering. Age and growth estimation is then stated as the Bayesian selection of a subset of ring curves, which combines a measure of curve significativity and an a priori statistical growth model. Experiments on real samples demonstrate the efficiency of the proposed data extraction stage. Our Bayesian framework is shown to significantly outperform previous methods for the interpretation of a data set of 200 plaice otoliths and compares favorably with interexpert agreement rates (88\% of agreement to expert interpretations).
\end{abstract}

Keywords: otoliths de poissons , croissance ; âge ; analyse des images ; tissu biologique 


\section{Problem statement and related work}

Age and growth data provide key information for a broad range of scientific issues: for instance,

30 on the shell of sea scallops (Chauvaud 2004), the determination of age-length keys from the interpretation of fish otoliths for marine stock assessments (Panfili et al. 2003), the analysis of fish otolith growth patterns for marine ecology (Hare and Cowen 1997; Panfili et al. 2003) or tree-ring dating for archeology (Baillie 1982). All of these applications initially rely on the interpretation of growth rings which will be subsequently referred as biological hard tissues (Fig. 1). The presence of concentric ring structures is due to the variations in chemistry during the accretionary formation of these structures. The deposit of the rings is often associated with a biological periodicity (either seasonal or daily, depending on the species and the tissue). Counting growth rings then leads to an estimation of the age of rings (Fig. 2), however, not all the observed ring structures are relevant for aging (Campana 2001).

The previously mentioned biological applications require large collections of age and growth data for a consistent analysis. The acquisition of this data is generally performed by expert readers (typically, for marine stock assessment issues, several thousands of fish otoliths per year). The development Hirschhorn 1987; Guay 1997; Morison et al. 1998). Our work focuses on the automation of age and growth estimation from the analysis of ring structures within the images of biological hard tissues. As detailed below, the key issues lie in the use of growth information to design a ring extraction framework that takes into account growth non-linearity (Fig. 2.b), and to discriminate actual growth rings from irrelevant ones (Campana 2001). Our approach is tested on fish otoliths, which provide representative samples in terms of complexity of the interpretation task as well as a mean for a quantitative comparison to previous work (Welleman and Storbeck 1995; Troadec et al. 2000; Guillaud et al. 2002). 
There are two broad categories of approaches for the analysis of growth ring structures within the images of biological hard tissues. One the one hand, 1D (one-dimensional) methods restrict their analysis to an intensity profile which is extracted along a given reading axis. Different schemes have been proposed, using filtering techniques (Welleman and Storbeck 1995; Guay 1997) or time-frequency analysis (Lagardère and Troadec 1997; Fablet et al. 2003). The main drawback lies in the absence of 2D (two-dimensional) perception of ring continuity, which makes the method sensitive to local artifacts resulting in the low aging performances reported in Guillaud etal. (2002). However, despite the large research effort devoted to the detection of ridges and valleys in generic images (Cohen 1991; McInerney and Terzopoulos 1996; Lindeberg 1998), more efficient algorithms are needed to achieve 2D ring segmentation in the images of biological hard tissues. Two main features need to be further investigated: the geometric properties of concentricity of the growth rings and the irregularity of image features due to the underlying non-linear growth pattern. Several solutions have been developed to 65 tackle these issues: deformable templates deduced from the external shape of the hard tissues (Troadec et al. 2000), a graph framework (Rodin et al. 1996), locally deformable spline-based models (Benzinou et al. 1997) and multi-agent systems (Guillaud et al. 2002). The quantitative evaluation carried out for fish age estimation in Guillaud etal. (2002) point out that important improvements were obtained compared to 1D approaches, however, important difficulties still remain. Whereas different 2D approaches search for closed 2D rings, growth rings are often only partially seen (Fig. 3). In addition, while these 1D and 2D techniques perform age and growth estimation by selecting all the detected rings, for validated ageing protocols, the interpretation step is a complex task (Campana 2001). Two main issues arise: the discrimination of actual growth rings from local artifacts, false rings or checks (Fig. 3), and the high biological variability, in terms of ring appearance and shape, as well as in growth patterns (Fig.

4). The presence of false rings is often due to biological or environmental events (for instance, sexual maturity, environmental stress, etc.). Both ring strength (in terms of image contrast), ring length and inter-ring distances are implicitly exploited by expert readers. However, using current ageing protocols, 
it remains difficult to define the objective and quantitative criteria of ring appearance and growth increment that permit actual growth rings to be distinguished from false rings or checks. These important issues demonstrate that only expert readers with a broad experience for given species and stocks can precisely and accurately estimate ages, since they have observed several tens of thousands of otoliths before becoming consistent in their interpretation. The difficulty in defining well-formalized criteria for the interpretation of growth rings has been emphasized by the results of a European workshop on plaice otolith reading held in 2003 (Easy 2003): inter-reader agreement rates range from $40 \%$ to $95 \%$ depending on the readers' experience for fish samples from age groups one to six, with between $85 \%$ and $95 \%$ agreement among expert readers. These results illustrate the complexity of the otolith interpretation even for "easy-to-read" samples.

Discriminating realistic growth patterns from irrelevant ones is a crucial step in designing an automated approach for fish age and growth estimation and was recently investigated using statistical learning (Robertson and Morison 1998; Fablet and Josse 2005). In both studies, age estimation is stated as a classification issue which resorts to mapping a vector of features of the content of otolith images (e.g., the vector formed by the Fourier coefficients of a 1D intensity signal along a given axis in Robertson and Morrison (1998)) to an age class. Although results appear promising results have been reported, these schemes only perform age estimation and cannot be extended to growth estimation. In particular, they cannot deliver an iconic interpretation of the image depicting the structures selected as the growth rings (for instance, by drawing markers on the image structures selected as growth rings (Fig. 3)).

To bring new solutions to these issues, a novel two-stage approach is developed in this paper. The first stage of this approach aims at extracting 2D growth rings within images of biological hard tissues. This segmentation issue is stated as the detection of concentric ridge and valley structures. Because the assumption that 2D growth rings are closed, as previously considered (Rodin et al. 1996; Troadec et al. 2000; Guillaud et al. 2002), is often violated, a bottom-up strategy is adopted, which initially 
extracts elementary ridge and valley segments. To ensure robust detection, a template-based scheme, based on the deformation of local templates issued from the external shape of the hard tissue, is cou-

ring curves, exploiting ring concentricity and the alternation of opaque and translucent rings. The second stage of the proposed technique tackles the actual interpretation of growth ring structures. Given candidate ring curves, age and growth estimation resorts to the selection of a relevant curve subset. The proposed Bayesian setting formally models the protocols followed by expert readers. Using the MAP (Maximum A Posteriori) criterion (Geman and Geman 1984), the proposed likelihood function combines both of the ignificativity measures evaluated for each extracted ring curve, a priori growth information to discriminate realistic growth patterns from irrelevant ones and hard constraints on the spatial arrangement of the selected ring curves. In particular, an a priori growth model is defined from examples of actual growth patterns. To our knowledge, this work is the first attempt to explicitly rely on experts' protocols and a priori growth information to constrain the automated interpretation of growth rings within images of biological hard tissues. The different steps of our approach for 2D ring segmentation and image interpretation are described and illustrated for a four year old plaice otolith (Fig. 5). The set of detected elementary ridge segments (Fig. 5b), the ridge curves resulting from the grouping of the elementary segments (Fig. 5c) and the selected subset of ridge curves (Fig. 5d).The parameter setting used to process this example is detailed below.

\section{Semi-local extraction of elementary ring segments}

\section{Local template-based framework}

As a result of the accretionary formation of biological hard tissues, the alternated translucent and opaque rings are concentric with respect to the growth center. In addition, ring shapes can be roughly be approximated (at least locally) as scaled versions of a given template. This key feature was exploited in Benzinou etal. (1997), where the detection of closed 2D rings is reliant on the scaling of the external 
shape of the hard tissue relative to the growth center. While promising, this technique results in poor detection of the rings close to the edge. This is partly due to the sensitivity of this template-based approach to the accuracy of locating the growth center, as well as to ring discontinuities. On the other hand, purely local techniques for ridge and valley detection (Haralick 1983; Chalmond et al. 1995; Lindeberg 1998) are outperformed by parametric ones if accurate parametric models of ridge and valley shapes within the processed images are available.

Hence, the use of the external shape as the parametric model of ridge and valley structures is further investigated, and the global template approach is extended to a semi-local framework. Within predetermined angular sectors, scaled versions of a semi-local template model are fitted to local ridge or valley image segments (Fig. 6). The semi-local template model is still derived from the external shape. This semi-local parametric framework is a good compromise between the ability to adapt to local ridge and valley shape, and the resistance to noise within images. In particular, as shown below, it is more resistant to errors in identifying growth centers than the template-based approach described in Troadec etal. (2000), which permits the use of algorithms aimed at the automatic detection of the growth center (Guillaud et al. 2002; Cao and Fablet 2004).

More precisely, the extraction of local elementary ridge or valley segments proceeds as follows. Given an image and the position $\left(x_{O}, y_{O}\right)$ of the growth center $O$, its external shape is initially extracted. The number of sampling points of the external shape is denoted by $N_{C}$ and their polar coordinates by $\left\{\left(\rho_{C}^{k}, \theta_{C}^{k}=2 k \pi / N_{C}\right)\right\}_{k \in\left[1, N_{C}\right]}$, in the framework of the two principal axes centered in the growth center. The semi-local detection of ridge and valley structures is performed within $N_{S L}$ overlapping angular sectors, referenced by their angular coordinate $\left\{\theta_{S L}^{n}=2 n \pi / N_{S L}\right\}_{n \in\left[1, N_{S L}\right]}$ and their angular width $2 * \Delta \theta_{S L}$. To ensure overlapping of the angular sectors, $\Delta \theta_{S L}$ is set such that $\Delta \theta_{S L}>\pi / N_{S L}$. Given an angular sector $\theta_{S L}^{n} \pm \Delta \theta_{S L}$, the associated semi-local template model is defined by its polar coordinates $\left\{\left(\rho_{C}^{k}, \theta_{C}^{k}=2 k \pi / N_{C}\right)\right\}_{k \in \mathcal{K}^{n}}$.

Our goal is to determine the scaling factors $\alpha \in[0,1]$ such that the scaled template, defined by (C)2005 NRC Canada 
the polar coordinates $\left\{\left(\alpha \rho_{C}^{k}, \theta_{C}^{k}\right)\right\}_{k \in \mathcal{K}^{n}}$ with $\mathcal{K}^{n}=\left\{\left[1, N_{C}\right] /\left|\theta_{C}^{k}-\theta_{S L}^{n}\right|<\Delta \theta_{S L}[2 \pi]\right\}$, fit the local ridge and valley segments within the angular sector $\theta_{S L}^{n} \pm \Delta \theta_{S L}$. Different features computed from the derivatives of the intensity function along and normal to the local template (Lindeberg 1998) can

$$
s_{S L}^{n}(\alpha)=\operatorname{med}\left(\left\{I\left(x_{0}+\alpha \rho_{C}^{k} \cos \theta_{C}^{k}, x_{0}+\alpha \rho_{C}^{k} \sin \theta_{C}^{k}\right)\right\}_{\left\{k \in \mathcal{K}^{n}\right.}\right)
$$

where $\operatorname{med}()$ is the median estimator and $I$ the image intensity function. The extraction of the elementary ridge and valley segments then resorts to the detection of the extrema of the intensity function

(2) $\quad \frac{d s_{S L}^{n}(\alpha)}{d \alpha}=0$

\section{Ridges and valleys detection}

Solving for Eq2 requires computing the derivatives $d s_{S L}^{n}(\alpha) / d \alpha$ at relevant scales (Lindeberg 1998). In addition, specific time-frequency features of the images of biological hard tissues have to be considered. First, smooth variations of material density (for instance, otolith are usually thicker close to the edge) might result in a continuous evolution of the mean intensity values from the growth center to the edge. Second, the width of translucent and opaque rings is directly related to the underlying growth pattern. Growth patterns are mainly non-linear. Images of biological hard tissues then involve a frequency modulation applied to the ring structures. The actual growth pattern is obviously unknown, but a mean a priori growth model can be used for demodulation purposes. This idea was 
mean growth model is derived from the growth patterns associated to images interpreted by an expert. Mathematically, the mean growth model is defined by the function $L=\Phi(t)$, where $L$ is the distance to the otolith center, and $t$ the time variable in years.

The framework used to solve for Eq2 then initially demodulates $s_{S L}^{n}$ w.r.t. the growth model $\Phi$. The resulting demodulated signal $s_{D M}^{n}(t)$ is given by:

$$
s_{D M}^{n}(t)=s_{S L}^{n}\left(\frac{\rho_{C}^{n}}{\rho_{C}^{n^{*}}} \Phi(t)\right)
$$

where $n^{*}$ refers to the point of the external shape on the main reading axis (for instance, $\theta_{C}^{n^{*}}=\pi$ for plaice otoliths). Let us point out that $\Phi(t)$ is normalized w.r.t. $\rho_{C}^{n^{*}}$ since the mean growth model is learned along the main reading axis.

To remove the trend $s_{T D M}^{n}$ from the intensity signal $s_{D M}^{n}, s_{T D M}^{n}$ is estimated using a convolution to a Gaussian kernel $g_{\sigma_{T D M}}$ with a large variance $\sigma_{T}^{2}: s_{T D M}^{n}=g_{\sigma_{T}} * s_{D M}^{n}$. Then, the detection of the positions of the local ridges and valleys involves the computation of the derivative $d_{t} s_{D M}^{n}$ of the intensity function $s_{D M}^{n}-s_{T D M}^{n}$. $d_{t} s_{D M}^{n}$ is estimated using a Gaussian kernel $g_{\sigma_{D}}$ with a low variance $\sigma_{D}^{2}$

$$
d_{t} s_{D M}^{n}=\frac{d\left[g_{\sigma_{D}} *\left(s_{D M}^{n}-s_{T D M}^{n}\right)\right]}{d t} .
$$

To actually determine the extrema of $s_{D M}^{n}-s_{T D M}^{n}$, the zero crossings of its first derivative $d_{t} s_{D M}^{n}$ are detected for time values uniformly sampled within $\left[0, t_{\max }\right]$, with $t_{\max }$ computed such that $\Phi\left(t_{\max }\right)=\rho_{C}^{n^{*}}$. The scaling factors solving for Eq2 are then computed from the inverse transform $\Phi^{-1}$. Typically, the sampling rate is set to $0.01, \sigma_{D}$ to 0.1 and $\sigma_{T D M}$ to 1.5 (all these values are given in years since they are used in the demodulated domain).

The detection of the elementary ridge and valley segments arose from the adapted filtering of the semi-local template-based function $s_{S L}^{n}$ is shown (Fig. 7). This example stresses that the growth- 
adapted demodulation eases the detection of ridge and valley structures close to edge and avoids overdetections close to the center. Applied to the whole set of angular sectors $\left\{\theta_{S L}^{n}=2 n \pi / N_{S L}\right\}_{n \in\left[1, N_{S L}\right]}$ , the growth-adapted framework finally results in the detection of elementary ridge and valley segments (Fig. 5b).

195

\section{Extraction of 2D ridge and valley curves}

Given the set of extracted elementary ridge and valley segments (as depicted in Fig. 5), the aim is to merge these elementary segments to form meaningful curves. Numerous perceptual grouping algorithms have been developed to form segments from candidate points or elementary segments (Guy and Medioni 1996; Jacobs 1996; Cao 2003). Mainly, these techniques rely on general geometric criteria such as good continuations (Cao 2003) or curve smoothness (Guy and Medioni 1996; Jacobs 1996). When dealing with the analysis of growth rings within hard tissue images, three geometric features are of key interest: the growth rings are concentric, there is an alternation of opaque and translucent rings, opaque and translucent rings never cross each other.

The proposed grouping procedure exploits these features. More precisely, it proceeds as follows for elementary ridge segments (and, conversely for valley ring structures). For a given pair of elementary ridge segments associated to two neighboring angular sectors $\left(\theta_{S L}^{n}, \Delta \theta_{S L}\right)$ and $\left(\theta_{S L}^{n+1}, \Delta \theta_{S L}\right)$, the decision rule used to merge this pair of segments evaluates whether or not elementary valley segments within the neighboring angular sectors $\left\{\left(\theta_{S L}^{k}, \Delta \theta_{S L}\right)\right\}_{k \in\{n-1, \ldots, n+2\}}$ lie between the pair of tested segment in terms of distance to the growth center. This decision rule is illustrated (Fig. 8) for two cases: one where the merging is validated and the other one where it is rejected. A ridge curve is finally formed by the set of elementary ridge segments which can be linked by a path going through merged segments.

An example of the extraction of ridge curves from a set of elementary segments is displayed (Fig. 5c). It should be pointed out that the length of resulting curves depend both on the actual length of the 
215 rings present in the image, and on noise or local occlusions in the image. Depending on these features, the extracted ring curves can be closed curves as well as only partial ring curves.

\section{Image interpretation for age and growth estimation}

From a set of extracted 2D ridge and valley curves, the actual interpretation of the image content in terms of age and growth estimation depends on the selection of a curve subset corresponding to the actual growth rings. Several curve subsets might correspond to the correct interpretation, since an actual growth ring might be covered by several partial curves, and some detected curves might not correspond to actual rings, but to false rings, or due to noise patterns. The proposed framework relies on the protocols followed by expert readers. Mainly, it involves four different aspects: the focus on an a priori image region around a predefined axis, along which the considered images are known to convey more reliable and more relevant information; the detection of candidate ring-like structures; the evaluation of the significativity of any possible rings with respect to length and contrast information; the analysis of the spatial arrangement of the selected ring structures, which needs both to correspond to an alternation of translucent and opaque rings, and to conform to some a priori growth features.

To satisfy these protocols, a mathematically-sound Bayesian framework is developed. The associated data-driven term evaluates the significativity of the extracted candidate ring segments, whereas the a priori model integrates both some knowledge on growth variability as well as hard constraints set on the spatial arrangement of the selected rings. As pointed out previously, the discrepancies in disagreement rates in the interpretations of hard tissues for validated ageing protocols prove that it is crucial to benefit from the experience of expert readers (Easy 2003; Panfili et al. 2003). The key point is to define a relevant representation of the a priori growth information, which is addressed by deriving our a priori growth model from examples of actual growth patterns estimated by experts.

Our Bayesian setting states the selection of a relevant curve subset as a labeling issue, where each extracted ridge or valley curve is assigned a binary label, "selected" or "rejected". Let $\mathcal{C}$ denote the set 
of extracted ridge and valley curves as described in the previous section. Let $\mathcal{L}$ be the binary label set $\{$ "selected", "rejected" $\}, e_{\mathcal{C}}$ the set of labels $\left\{e_{c}\right\}_{c \in \mathcal{C}}$ for a given labeling configuration, $o_{\mathcal{C}}=\left\{o_{c}\right\}_{c \in \mathcal{C}}$ the features computed for all ring curves $c \in \mathcal{C}$ to evaluate their significativity. The selection of the relevant curve subset then comes to retrieve the best labeling configuration $\hat{e}_{\mathcal{C}}$ maximizing the MAP criterion:

$$
\hat{e}_{\mathcal{C}}=\arg \max _{e_{\mathcal{C}} \in \mathcal{L}^{|\mathcal{C}|}} P\left(e_{\mathcal{C}} \mid o_{\mathcal{C}}\right)=\arg \max _{e_{\mathcal{C}} \in \mathcal{L}^{|\mathcal{C}|}} P\left(o_{\mathcal{C}} \mid e_{\mathcal{C}}\right) P\left(e_{\mathcal{C}}\right)
$$

where $P\left(o_{\mathcal{C}} \mid e_{\mathcal{C}}\right)$ is the data-driven term described in the subsequent and $P\left(e_{\mathcal{C}}\right)$ integrates an a priori knowledge on growth variability and on the spatial arrangement of the relevant labeling configurations. Given $g_{e_{\mathcal{C}}}$ the growth pattern associated to the label configuration $e_{\mathcal{C}}$ (i.e., the growth pattern computed for the selected ring curves $S\left(e_{\mathcal{C}}\right)\left\{c^{*} \in \mathcal{C} / e_{c^{*}}=\right.$ selected $\}$, and $\Theta_{S\left(e_{\mathcal{C}}\right)}$ the set of angular sectors covered by the selected ring curves $S\left(e_{\mathcal{C}}\right)$, the a priori model $P\left(e_{\mathcal{C}}\right)$ is formally defined by:

$$
P\left(e_{\mathcal{C}}\right)=P\left(g_{e_{\mathcal{C}}}, \Theta_{S\left(e_{\mathcal{C}}\right)}\right)=P\left(g_{e_{\mathcal{C}}} \mid \Theta_{S\left(e_{\mathcal{C}}\right)}\right) P\left(\Theta_{S\left(e_{\mathcal{C}}\right)}\right)
$$

The likelihood $P\left(g_{e_{\mathcal{C}}} \mid \Theta_{S\left(e_{\mathcal{C}}\right)}\right)$ measures the relevance of the growth pattern $g_{\mathcal{E}_{\mathcal{C}}}$ w.r.t. a priori growth information. Since this model does not depend on direction information, $P\left(g_{e_{\mathcal{C}}} \mid \Theta_{S\left(e_{\mathcal{C}}\right)}\right)$ simplifies to $P\left(g_{e_{\mathcal{C}}}\right)$. As a complementary stage, the second term $P\left(\Theta_{S\left(e_{\mathcal{C}}\right)}\right)$ evaluates whether or not the considered labeling configuration conforms to the hard constraints set on the spatial arrangement of the ring curves.

Our Bayesian setting finally comes to solve for:

$$
\hat{e}_{\mathcal{C}}=\arg \max _{e_{\mathcal{C}} \in \mathcal{L}^{|\mathcal{C}|}} P\left(o_{\mathcal{C}} \mid e_{\mathcal{C}}\right) \cdot P\left(g_{e_{\mathcal{C}}}\right) \cdot P\left(\Theta_{S\left(e_{\mathcal{C}}\right)}\right)
$$

Further details regarding the associated likelihood functions are given in the subsequent. 


$$
P\left(o_{\mathcal{C}} \mid e_{\mathcal{C}}\right)=\prod_{c \in \mathcal{C}} P\left(o_{c} \mid e_{c}\right)
$$

As previously mentioned, experts mainly rely on the perception of contrast and ring continuity. Since contrast information is already implicitly used to extract ring curves, the likelihood $P\left(o_{c} \mid e_{c}\right)$ is defined from the angular length $L_{c}$ of the curve $c$. Using an energy setting, $P\left(o_{c} \mid e_{c}\right)$ is expressed as: $P\left(o_{c} \mid e_{c}\right) \propto \exp \left[-U_{1}\left(L_{c}, e_{c}\right)\right]$. The energy function $U_{1}$ favors the selection of ridge and valley curves with a high length value:

$$
\left\{\begin{array}{ll}
U_{1}\left(L_{c}, e_{c}\right)=1-s_{1}\left(L_{c}-\nu_{1}\right), & \text { if } e_{c}=\text { "selected" } \\
U_{1}\left(L_{c}, e_{c}\right)=s_{1}\left(L_{c}-\nu_{1}\right), & \text { if } e_{c}=\text { "rejected" }
\end{array},\right.
$$

where $s_{1}()$ is a smooth step function rescaled between 0 and 1 and $\nu$ the length threshold. This parameter value is easy to set since it simply expresses from which length value a ring curve is regarded as visually significant. Typically, this significativity threshold in terms of angular length is set to $\pi / 8$ (in radian).

\section{A priori growth information}

The interpretation of growth rings on hard tissues implicitly relies on growth information, although expert readers do not generally exploit explicit measurements. As highlighted for plaice otolith samples (Fig. 4), biological growth phenomena usually involve a very high variability. For instance, for this dataset, the distance between the first translucent ring and the growth center is between $0.7 \mathrm{~mm}$ and 2.7 $\mathrm{mm}$, while this distance for the second translucent ring is between 2.2 and $5 \mathrm{~mm}$. This huge overlapping 
between successive year classes prevent the use of a mean growth model to predict the position of each translucent ring. More complex representations are therefore required. Besides, in our Bayesian setting, labeling configurations $\left\{e_{\mathcal{C}}\right\}$ can correspond either to actual or to erroneous growth patterns. Consequently, rather than determining an accurate representation of the statistical distribution of the growth patterns for each year class, one is interested in designing a decision function which permits discrimination between realistic and irrelevant growth patterns.

An exemplar-based strategy is adopted to tackle this pattern recognition issue. It is assumed that a set of growth patterns representative of the actual biological variability is available. Let us denote by $\left\{g_{i}\right\}_{i \in\{1, . ., n\}}$ the set of $n$ examples of growth patterns for the year class $k$. The a priori growth model is then defined from the lower and upper envelopes of $\left\{g_{i}\right\}_{i \in\{1, . ., n\}}$ and of their derivatives. The lower envelope $g_{\min }^{k}$ is given by:

$$
g_{\min }^{k}(t)=\min _{i \in\{1, . ., n\}} g_{i}(t), \text { for } t \leq k,
$$

and the upper one $g_{\max }^{k}$ is given by:

$$
g_{\text {max }}^{k}(t)=\max _{i \in\{1, . ., n\}} g_{i}(t), \text { for } t \leq k,
$$

where $t$ is the time variable in years. Similarly, the lower and upper envelopes $d g_{\min }^{k}$ and $d g_{\max }^{k}$ of the first-order derivative of the growth functions $\left\{g_{i}\right\}$ are computed. The envelope of the firstorder derivatives is considered, since growth increments also provide meaningful cues to characterize realistic growth patterns. The computation of these envelopes for a set of examples of plaice growth patterns for the year class $k=4$ is depicted (Fig. 9). These min/max features are often implicitly used by expert readers to check the reliability of growth increments given their knowledge of growth variability.

From these envelopes, a probabilistic decision function $P\left(g_{e_{\mathcal{C}}}\right)$ is designed to evaluate whether (c)2005 NRC Canada 
or not the growth pattern $g_{e_{\mathcal{C}}}$ associated to a given labeling configuration $e_{\mathcal{C}}$ is relevant. More precisely, the labeling configuration $e_{\mathcal{C}}$ is assumed to refer to a realistic growth sample if $g_{e_{\mathcal{C}}}$ and $d g_{e_{\mathcal{C}}}$ respectively remain within the variability range defined by $\left(g_{\min }^{k}, g_{\max }^{k}\right)$ and $\left(d g_{\min }^{k}, d g_{\max }^{k}\right)$. Fig. 10 provides an illustration of this statement. Formally, $P\left(g_{e_{\mathcal{C}}}\right)$ is written as:

$$
P\left(g_{e_{\mathcal{C}}} \text { is relevant for age class } k\right)=\min \left(P\left(g_{e_{\mathcal{C}}}, g_{\min }^{k}, g_{\max }^{k}\right), P\left(d g_{e_{\mathcal{C}}}, d g_{\min }^{k}, d g_{\max }^{k}\right)\right)
$$

where $P\left(g_{e_{\mathcal{C}}}, g_{\min }^{k}, g_{\max }^{k}\right)$ is defined by:

$$
P\left(g_{e_{\mathcal{C}}}, g_{\text {min }}^{k}, g_{\text {max }}^{k}\right)=\min \left[\min _{0 \leq t \leq k} s_{2}\left(g_{e_{\mathcal{C}}}(t)-g_{\text {max }}^{k}(t)\right), \min _{0 \leq t \leq k} 1-s_{2}\left(g_{e_{\mathcal{C}}}(t)-g_{\min }^{k}(t)\right)\right]
$$

with $s_{2}$ is the opposite of a smooth step function rescaled between 0 and 1. $P\left(d g_{e_{\mathcal{C}}}(t), d g_{\text {min }}^{k}, d g_{\text {max }}^{k}\right)$ is defined in the same way. The introduced growth model can be viewed as a binary classifier (realistic vs. non-realistic) of growth patterns defined by the probabilistic decision function $P\left(g_{e_{\mathcal{C}}}\right)$.

The evaluation of the likelihood $P\left(g_{e_{\mathcal{C}}}\right.$ is relevant for age class $\left.k\right)$ requires normalizing the growth pattern $g_{e_{\mathcal{C}}}$ w.r.t. the reference growth axis exploited to estimate $g_{\min }^{k}, d g_{\max }^{k}, d g_{\min }^{k}$ and $g_{\max }^{k}$. This normalization issue comes to define the normalized positions of the extracted ring curves in $\mathcal{C}$ along the reference growth axis. It is iteratively performed as follows. Ring curves in $\mathcal{C}$ intersecting the reference axis initially form the set of curves $\mathcal{C}_{\text {Norm }}$ with known normalized positions. Iteratively, the curve $c^{*}$ in $\mathcal{C} \backslash \mathcal{C}_{N o r m}$ the closest to the reference axis in the direction normal to the axis is selected. The two curves $\left(c_{1}, c_{2}\right)$ in $\mathcal{C}_{N o r m}$, angularly overlapping $c^{*}$ and the closest to $c^{*}$, are then retrieved, and the normalized position of $c^{*}$ is lineally interpolated from the normalized positions of $c_{1}$ and $c_{2}$. This normalization procedure does not assume neither an assumption that the reference axis is a straight line nor that the growth is angularly isotropic. It can then be applied to complex growth situations, given that the reference growth axis can be automatically extracted for all samples. 


\section{Constraints on the spatial arrangement of rings}

In addition to a priori growth information, hard constraints on the spatial arrangement of rings are set to help in the minimization of $\mathrm{Eq}(7)$. These constraints are introduced in the proposed Bayesian

setting through the evaluation of the likelihood $P\left(\Theta_{S\left(e_{\mathcal{C}}\right)}\right)$. The likelihood is set to zero if any of the constraints described below are not verified. The three hard constraints are as follows. As mentioned previously, expert readers perform the interpretation of growth rings along a main reading axis, therefore, the analysis is restricted to a predefined angular sector of interest, and selected ring curves $S\left(e_{\mathcal{C}}\right)$ are required to intersect this angular sector. Any relevant interpretation $S\left(e_{\mathcal{C}}\right)$ must conform to the studied biological structures, which involve an alternation of translucent and opaque growth rings, consequently, $S\left(e_{\mathcal{C}}\right)$ is required to depict an alternation of valley and ridge curves. Examples are shown to illustrate this second hard constraint (Fig. 11). Finally,successive ring curves within $S\left(e_{\mathcal{C}}\right)$ must angularly overlap, which will lead to visually consistent interpretation.

\section{Energy minimization}

Given a curve set $\mathcal{C}$, the interpretation comes to solve for Eq7. Since $\mathcal{C}$ typically involves about 50 curves, one cannot test over the whole set of $2^{|\mathcal{C}|}$ possible configurations. One might stress that not all

of the $2^{|\mathcal{C}|}$ configurations are relevant ones. However, the number of relevant configurations cannot be determined analytically from $|\mathcal{C}|$, since it depends on the relative positions of the extracted curves. Its evaluation would require testing over all possible configurations. In practice, it has been determined that the total number of relevant configurations was greater than 1000 for about 50 extracted curves.

Consequently, the minimization issue is carried out in a causal manner by introducing one-by-one ring curves $c \in \mathcal{C}$ and retaining only the $N_{\max }$ best configurations w.r.t. likelihood values $P\left(e_{c} \mid o_{c}\right)$ (see as detailed in appendix). 


\section{Experiments}

For all the experiments reported in this Section, the same parameter setting is used. The external shape of the hard tissue is represented by $N_{C}=500$ points, and smoothed by a Gaussian kernel of variance 0.01 . The extraction of elementary ring segments is performed for $N_{S L}=75$ local axes sampled uniformly. The angular sector used for each local template is $\Delta \theta_{S L}=\pi / 90$. The growthadapted filtering is performed with $\sigma_{T}=1.5$ and $\sigma_{D}=0.05$ (these values are given w.r.t. time in years or in days depending on the periodicity of the observed ring structures). Concerning the Bayesian labeling of the set of extracted ring curves, the analysis is restricted to an angular sector of $\mathcal{S}=$ $\{\theta,|\theta-\pi| \leq \pi / 8[2 \pi]\}$ around the first principal axis of the otolith. The threshold $\nu_{1}$ is set to $\pi / 8, \beta$ to 10 .

All images used in the experiments have been acquired with a $1000 \times 1000$ digital video camera under a binocular. A polarized light was used to better separate biological hard tissues from the background. No other preprocessing step was performed.

\section{Extraction of meaningful ring curves}

Three examples of the extraction of meaningful ring curves within images of biological hard tissues are first reported (Fig. 12): a plaice otolith, the shell of a bivalve seashell, and the section of a tree trunk. All these images share common characteristics in terms of rather concentric ridge and valley structures associated to a growth-based modulation. As expected, the proposed template-based growth-adapted scheme extracts meaningful ring curves mainly corresponding to the actual growth rings.

The extraction scheme is also applied to structures depicting ontogenetic changes in the shape of the rings (Fig. 13). Using a global template approach (Troadec et al. 2000), poor detection results are reported (Fig.14). On contrast, the proposed local template-based approach is able to successfully identify meaningful partial ring curves. It demonstrates first that fitting local scaled versions of the external shape provides a mean to adapt to non-radial growth. Second, it also stresses that growth- 
adapted detection of ridges and valleys only require a rough knowledge of the scales relevant to solve for Eq2. In other words, as used by the proposed demodulation scheme, the distance to the otolith center is a sufficient cue to approximate the width of the structures to be extracted.

\section{Interpretation examples}

To evaluate the performance of the proposed Bayesian interpretation scheme, fish otolith analysis is emphasized. The design of the a priori growth model requires building a large growth data collection (typically, several hundreds of samples). Our evaluation set consists of 400 plaice otoliths from the age determination from plaice otoliths has been validated and confirmed (Campana 2001; Easy 2003). The processed otolith collection does not contain known-age material, but has been interpreted and annotated by an expert from Ifremer according to the agreed and validated plaice ageing protocol (Easy 2003). All the images have been acquired using transmitted light with the same magnification. Half of these interpretations are used to train the a priori growth model, and the other half are used as a control for the evaluation of our approach.

Three images of plaice otoliths have been chosen to illustrate the behavior of the different stages of our approach (Fig. 15): the first two belong to the age group six (seven translucent rings) and the third one to the age group four (five translucent rings). For these three examples, the age and growth estimation was performed by an expert reader, whose interpretation is used as the control. Reported results include both the extracted ridge curves, the selected ridge curve subsets using the Bayesian framework, and the estimated growth pattern compared to the control.

The first example is a six year old plaice otolith (seven translucent rings). Relevant ridge curves are extracted, and the selected interpretation is the same as the expert one. This example was chosen to illustrate the ability of our approach to extract ridge and valley structures close to the otolith edge thanks to the growth-adapted filtering used within the semi-local template framework. 
A plaice otolith also assigned to the age group six is considered for the second example. It emphasizes the need for an approach capable of extracting non-closed curves, such as the second translucent ring. The proposed approach extracts meaningful ridge and valley curve both for closed and non-closed seasonal rings, and retrieve the correct otolith interpretation.

The third example points out the requirement for formulating the otolith interpretation as a selection/rejection issue of extracted candidate ridge and valley curves. The expert has regarded the second and third translucent rings as belonging to a single translucent area. Given an accurate extraction of ridge and valley curves, the use of a statistical growth model within the introduced Bayesian framework results in discarding configurations which select ridge curves for the two rings of the second translucent area. Compared to the expert interpretation, the selected subset of ridge curves only differ in the position of the second translucent area: the expert has taken the middle of this area, whereas the automated approach has selected a ridge curve associated to the third translucent ring. Therefore, the age is correctly estimated, and the growth patterns just show a minor difference at between years one and two, due to the position chosen for the second translucent ring.

Computational time for our approach under Matlab 7 (The Mathworks Inc, Natide, Massachussetts) for the extraction of $2 \mathrm{D}$ ring curves runs about 30 seconds on a $2.4 \mathrm{GHz}$ Pentium IV, whereas the Bayesian labeling needs a few seconds.

\section{Quantitative evaluation}

A quantitative evaluation of the proposed approach has been carried out using the second half of the collected set of plaice otoliths (i.e., a database of 200 otoliths). The age distribution of this database is reported (Fig. 16a). Age groups one and two are the most frequent with more than fifty elements, whereas age groups five and six just comprise fifteen and twenty samples. This age distribution is representative of the estimates from commercial landings, where samples of age group six and less usually represent more than $90 \%$ of the total. Otoliths older than six can also be found, however, the 
number of samples is not sufficient to train a relevant growth model. Consequently, they were not considered during our evaluation.

The interpretations provided by the expert are used as the control to compute the class-by-class and overall age estimation errors (results are given in Fig. 16). The agreement rate is comprised of between

\section{Sensitivity to errors in pointing the growth center} center, the results of the extraction of ridge curves are reported for two different positions of the nucleus: the actual position of the growth center (Fig.17a) and a position given by the translation of $0.4 \mathrm{~mm}$ to the left and 0.25 upward of the actual growth center (Fig. 17b). Very few differences are observed in the accuracy of the detection of meaningful ring curves. 
To carry out a quantitative evaluation of the robustness to errors in locating the growth center, the effect of using a biased position of the growth center on age estimations has been investigated for the set of 200 plaice otoliths. Fewer than 5\% of the images are interpreted differently than with the actual position of the growth center (given the translated position of the growth center). These results stress the robustness of the proposed scheme to errors in locating otolith nucleus, given that the automated techniques for nucleus detection (Guillaud et al. 2002; Cao and Fablet 2004) were shown to perform with a squared-root variance below $0.15 \mathrm{~mm}$.

\section{Subjectivity in age reading}

Age estimation errors mainly occur with otoliths, for which the interpretation is difficult even for experts. Two representative examples are reported (Fig. 18). The first one involves an otolith aged as two by the expert, whereas the age estimated by the automatic approach is three. These two estimations differ in the interpretation of the area close to the edge: the expert considers only one very large third ring, whereas the automatic approach detects and selects a third ridge curve and a fourth one. Both interpretations are realistic in terms of growth patterns, but the expert has considered that the last ring was not sufficiently contrasted to be selected. The second case is an otolith aged as five by the expert, and six by the Bayesian approach. These two interpretations differ in the selection of an additional translucent ring before the first translucent ring selected by the expert. Whereas this potential translucent ring is slightly less contrasted compared to other translucent rings, its selection results in a more likely growth pattern, hence, it remains difficult to favor one or the other of these two interpretations.

These two examples of disagreement in otolith interpretations point out the difficulty in balancing between the likelihood of the growth pattern, and the perceptibility of translucent and opaque rings. Expert interpretation remains subjective for complex cases. For instance, for routine activities, interexpert or intra-expert agreement rates in age reading greater than $90 \%$ are considered as satisfactory. In addition, inter-expert agreement rates range between $85 \%$ and 95\% (Easy 2003), which demonstrates 
the relevance of the Bayesian approach for the automated interpretation of growth ring structures. It also demonstrates that this scheme can be used for routine ageing, either as an alternative to expert readings or as a low-cost double reading for verification purposes.

\section{Discussion and future work}

In this paper a new technique has been presented for the segmentation of 2D ridge and valley curves applied to the analysis of growth ring structures within images of biological hard tissues. It relies on a semi-local template-based scheme using a priori information on ring structures to detect ridges and valleys from an adapted filtering. The Bayesian selection of the extracted ridge and valley curves is proposed to perform the actual interpretation of the growth structures. The considered Bayesian modeling combines a measure of curve significativity and a statistical model of individual growth patterns of the considered species.

Good performances have been reported for the extraction of meaningful ring curves within images of biological hard tissues. Giving the emphasis on the interpretation of fish otolith images, the quantitative evaluation carried out for plaice samples stress that the proposed Bayesian framework significantly outperform previous work. The agreement rate w.r.t. expert interpretations can be favorably compared to inter-expert agreement rates, which demonstrates the feasibility of using this Bayesian scheme for routine analysis. Different issues then arise. First of all, a fully automated scheme requires combining the technique proposed in this paper to the automation of the acquisition of otolith images and of the detection of the otolith nucleus as proposed in Cao and Fablet (2004). For routine aging, the cost-benefits analysis of such automated systems need to balance the accuracy of the system compared to readers vs. the gain in terms of processing time. As stressed by our results, for an "easy-to-read" species, such as plaice, the potential gain is huge, since the accuracy levels of the automated system should be in the range of the inter-expert agreement rates, and greater than those observed for in-training readers. Even if the proposed automated system consider only the youngest age groups (up to 6), they represent more 
than $90 \%$ of the aged data for the considered species and stock. Hence, the use of the proposed scheme for the most important age groups should highly reduced the workload of expert readers. Since the proposed Bayesian scheme exploits an a priori growth model, its robustness in spite of variations in growth patterns should be investigated. The considered min/max model is potentially more stable over time than a mean model. This aspect is also highly species-dependent since not all species are known to show high dynamics in terms of growth patterns. In any case, the growth model could be trained on a yearly basis on about one or two hundred samples to reduce the number of samples to be interpreted 485 by the expert (by a factor of two or more).

Apart from routine aging, the proposed approach can be used in a number of different ways to assist the experts in the acquisition of age and growth data. As an illustration, one could use it as a low cost solution for a second reading of the samples to detect unrealistic interpretation or to evaluate confidence measures of the age estimation (Fablet 2005). Such measures are of great interest for assessment models (Reeves 2003). Another potential application consists of helping in the acquisition of the interpretation: given the age estimate provided by the expert, the Bayesian system could detect the most likely ring positions. These examples stress that the proposed system is not strictly limited to the automation of age and growth estimation, but it can bring a wide range of new computer-assisted tools for aging issues in the context of quality assurance and quality control (Morison et al. 1998).

In future work, the application of the proposed scheme to more complex structures and longer-lived species will be investigated. Our approach involves two main steps: the extraction of ring structures and the Bayesian interpretation step. The latter is generic, given that a subset of candidate ring curves is initially extracted. Therefore, it provides a strong basis for an application to more complex situations. Potential improvements mainly concern the statistical growth model. The use of statistical learning techniques (Fablet and Josse 2005) could be investigated. Besides, for longer-lived species or microstructures analysis, model training needs to be adapted to deal with a large number of age classes. On the other hand, the extraction step is a challenging task for highly complex structures. The two 
examples reported for a pollock and a cod otolith stress the ability of our approach to cope with midlevel complexity structures. Improvements could enable the analysis of highly complex samples (e.g., hake or tunus otoliths): for instance, from the estimation of the local direction of the growth rings in the images. As well, whereas the analysis of annual growth rings usually relies on the analysis of the hard tissue in a single focal plane, the interpretation of daily increments often requires observing the ring structures in several focal planes. To this end, one might rely on the analysis of the sequence of images acquired in successive focal planes. An additional fusion scheme will then be needed either to stack this sequence of images into a single image using the magnitude of the image gradient or to combine the results of the extraction step carried out within each image of the sequence. In both cases, the semi-local template-based approach presented in this paper would be a fundamental block.

\section{Acknowledgments}

The author wishes to thank André Ogor from Ifremer/LASAA for his help in acquiring the image sets, Marie-Line Manten, Ifremer expert, for providing the set of plaice otoliths and the associated their interpretations, and Abdeslamm Benzinou for fruitful discussion on the template-based approach. 


\section{References}

Baillie, M. 1982. Tree-Ring Dating and Archaeology. The University of Chicago Press, Chicago, Illinois.

Benzinou, A., Troadec, H., Bihan, J. L., and Rodin, V. 1997. The locally deformable B-Bubble model: an application to growth ring detection on fish otoliths. In Proc. of th 10th Scand. Conf. on Image Analysis, SCIA'97, pages 181-187, Lappeenranta, Finland.

Campana, S. 2001. Accuracy, precision and quality control in age determination, including a review of the use and abuse of age validation methods. J. Fish Biology, 59:197:242.

Cao, F. 2003. Good continuations in digital image level lines. In Proc of IEEE Int. Conf. on Computer Vision, ICCV'03, pages 440-447, Nice, France.

Cao, F. and Fablet, R. 2004. Automatic morphological detection of otolith nucleus. In Proc. of 17th Int. Conf. on Pattern Recognition, ICPR'04, volume 3, pages 606-609, Cambridge, UK.

Chalmond, B., Azencott, R., and Colefy, F. 1995. Markov fusion of a pair of noisy images to detect intensity valleys. Int. J. Comp. Vis., 16:135-145.

Chauvaud, L. 2004. The shell of the Great Scallop Pecten maximus as a tool for high frequency paleoenvironmental reconstruction. Paleooceanograpgy. Submitted.

Cohen, L. 1991. On active contour models and balloons. Comp. Vis., Gr. Im. Pr.: Im. Und., 53(2):211-218.

Easy, M. 2003. Report of the ICES workshop on plaice age determination. ICES, Ostende, Nethrelands, May 2003.

Fablet, R. 2005. Statistical learning for fish age and growth analysis from otolith images. Mar. Fres. Res. To appear.

Fablet, R. and Josse, N. L. 2005. Automated fish age estimation from otolith images using statistical learning. Fish. Res. 72(2-3): 279-290.

Fablet, R., Benzinou, A., and Doncarli, C. 2003. Robust time-frequency model estimation in otolith images for fish age and growth analysis. In Proc. of 10th Int. Conf. on Image Processing, ICIP’03, volume 2, pages 593-596, Barcelona, Spain.

Geman, S. and Geman, D. 1984. Stochastic relaxation, Gibbs distribution and the Bayesian restoration of images. IEEE Trans. Pat. An. Mach. Int., 6(6):721-741. 
Guay, R. 1997. Morphing technology applied to digital tree ring density measurement. In Int. Union of Forestry Org., Forest Products for Sustainable Forestry, Pullman, WA.

Guillaud, A., Benzinou, A., Troadec, H., Rodin, V., and Bihan, J. L. 2002. Autonomous agents for edge detection and continuity perception on otolith images. Im. Vis. Comp., 20(13-14):955-968.

Guy, G. and Medioni, G. 1996. Inferring global perceptual contours from local features. Int. J. Comp. Vis., 20(1):113-133.

Haralick, R. 1983. Ridges and valleys on digital images. Comp. Vis., Gr. Im. Pr., 22:28-38.

Hare, J. and Cowen, R. 1997. Effect of age, growth rate, and ontogeny on the otolith size - fish size relationship in bluefish, pomatomus saltatrix, and the implications for back-calculation of size in fish early life history stages. Can. Jal of Fish. Res. and Aqu. Sc., 52:1909-1922.

Jacobs, D. 1996. Robust and efficient detection of salient convex groups. IEEE Trans. Pat. An. Mach. Int., 18(1):23-37.

Lagardère, F. and Troadec, H. 1997. Age estimation in common sole solea solea larvae: validation of daily increments and evaluation of a pattern recognition technique. Mar. Ecol. Prog. Ser., 155:223-237.

Lindeberg, T. 1998. Edge detection and ridge detection with automatic scale selection. Int. J. Comp. Vis., 30(2):117-154.

McInerney, T. and Terzopoulos, D. 1996. Deformable models in medical images analysis: a survey. Med. Im. An., 1(2):91-108.

Morison, A., Robertson, S., and Smith, D. 1998. An integrated system for production fish aging: image analysis and quality insurance. N. Am. J. Fish. Man., 18:587-598.

Ogor, A. and Fablet, R. 2004. Tnpc software: digital processing of calcified structures. www.ifremer.fr/lasaa.

Panfili, J., Pontual, H. D., Troadec, H., and Wright, P. 2003. Manual of Fish Sclerochronology. Eds Ifremer.

Reeves, S. 2003. A simulation study of the implications of age-reading errors for stock assessment and management advice. ICES J. Mar. Sc., 60:314-328.

Robertson, S. and Morison, A. 1998. Development of an artificial neural network for automated age estimation. Technical Report 98/105, Marine and Freshwater Resources Institute.

Rodin, V., Troadec, H., Pontual, H. D., Bihan, J. L., Benzinou, A., and Tisseau, J. 1996. Growth ring 
detection on fish otoliths by a graph construction. In Proc. of 3rd IEEE Int. Conf. on Image Processing, ICIP'96, volume 2, pages 685-688, Lausanne, Switzerland.

Small, G. and Hirschhorn, G. 1987. Computer-assisted age and growth pattern recognition of fish scales using a digitizing tablet. In R.C. Summerfelt, G. H., editor, Age and Growth of Fish, pages 397-410. ISU Press. growth ring detection by digital image processing. Fish. Res., 46(1-3):155-163.

Welleman, H. and Storbeck, F. 1995. Automatic ageing of Plaice otoliths by means of image analysis. In Secor, D., Dean, J., and Campana, S., editors, Recent developments in Fish Otolith Research, pages 271-282. Univ. of South Carolina Press. ???? ,South Coralina. 
580

\section{Appendix A}

The algorithm ussed to solve for the minimization issue (7) proceeds proceeds as follows:

- Step 1:

1. $k=0$

2. Sort curves within $\mathcal{C}$ according to their normalized distance to the nucleus: $\mathcal{C}=\left\{c_{1}, . ., c_{|\mathcal{C}|}\right\}$

3. Initialize the set of current label configurations $\mathcal{E}^{0}$ to the empty set

\section{- Step 2:}

1. $k=k+1$

2. Define $\mathcal{C}^{k}=\left\{c_{1}, . ., c_{k}\right\}$

3. For any $e_{\mathcal{C}^{k-1}}$ within the configuration set $\mathcal{E}^{k-1}$, add the two new labeling configurations

- Step 3: repeat step 2 until $k=|\mathcal{C}|$
$\left\{e_{\mathcal{C}^{k-1}}, e_{k}=\right.$ "rejected" $\}$ and $\left\{e_{\mathcal{C}^{k-1}}, e_{k}=\right.$ "selected" $\}$ to the set of current configurations $\mathcal{E}^{k}$

4. Compute the likelihood values $P\left(o_{\mathcal{C}^{k}} \mid e_{\mathcal{C}^{k}}\right) \cdot P\left(g_{e_{\mathcal{C}^{k}}}\right) \cdot P\left(\Theta_{S\left(e_{\mathcal{C}^{k}}\right)}\right)$ for all the configurations $e_{\mathcal{C}^{k}} \in \mathcal{E}^{k}$

5. Sort these likelihood values

6. Keep only the $N_{\max }$ best configurations within $\mathcal{E}^{k}$

(C) 2005 NRC Canada 
Table 1. Accuracy of the estimation of the otolith growth pattern for the processed 200 plaice (Pleuronectes Platessa) otoliths from age classes 1 to 6: the mean values and the associated squared-root variances, with respect to the ring number, of the distances between the ring positions determined by the Bayesian scheme and those provided by expert interpretations, when the two age estimates agree, are evaluated.

\begin{tabular}{cccccccc}
\hline & 1st ring & 2nd ring & 3rd ring & 4th ring & 5th ring & 6th ring & 7th ring \\
\hline Mean value (mm) & 0.10 & 0.09 & 0.07 & 0.06 & 0.07 & 0.06 & 0.05 \\
Squared-root variance (mm) & 0.15 & 0.11 & 0.06 & 0.05 & 0.05 & 0.03 & 0.06 \\
\hline
\end{tabular}




\section{List of Figures}

1 Examples of images of biological hard tissues involving periodical ring structures exploited for age and growth analysis. (a) Image of the shell of a bivalve (spisule). Bar $=0.5 \mathrm{~cm}$; (b) image of a plaice otolith. Bar $=1 \mathrm{~mm}$; (c) image of the section of a tree

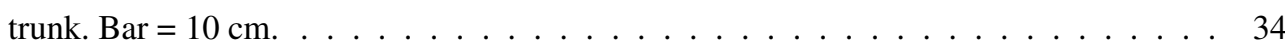

2 Illustration of plaice otolith interpretation for a 4 year old individual: (a) Image of a plaice (Pleuronectes Platessa) otolith acquired under a binocular with transmitted light. The interpretation of the winter translucent rings is displayed by the markers set on the radial drawn on the main reading axis. Bar $=1 \mathrm{~mm}$; (b) Associated growth pattern (distance from the otolith center to the last ring as a function of the age). . . . . . 35

3 Difficulties in otolith ring interpretation: this otolith presents false rings between the first and second rings, and between the second and the third ones. Besides, due to the non-linearity of the associated growth pattern, the last rings tend to be closer and hard to distinguish. The positions of validated rings is depicted by markers set on the radial drawn from the otolith center to the edge along the main reading axis. Bar $=1 \mathrm{~mm} . \quad \ldots \quad 36$

4 Illustration of biological growth variability for a set of plaice (Pleuronectes Platessa)

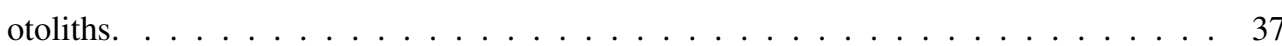

5 Illustration of our approach dedicated to the analysis of growth rings within images of biological hard tissues: (a) image of the observed hard tissue (here, a plaice (Pleuronectes Platessa) otolith), (b) extracted elementary "ridge" ring segments, (c) extracted "ridge" ring curves from the merging of elementary ring segments, (d) subset of the selected ridge ring curves using the developed Bayesian framework. Bar $=1 \mathrm{~mm} . \quad 38$ 
6 Local template-based scheme for the detection of elementary ring segments: (a) extraction of the external shape using polar coordinates in the framework of the two principal axis of the hard tissue and centered in the growth center $O$, (b) scaling of the local template for a given angular sector $\theta_{S L}^{n} \pm \Delta \theta_{S L} \ldots \ldots \ldots \ldots \ldots \ldots$

7 Detection of elementary ridge segments: (a) the processed image with the extracted elementary ridge segments (thick line) within the angular sector $\theta_{S}^{n} L=\pi($ Bar $=1$ $\mathrm{mm}$ ); (b) the intensity function $s_{S L}^{n}$, c) the demodulated version (red dashed line) using the mean growth law, the estimated tendency (blue dashed-dotted line), the filtered signal (magenta solid line) and the estimated extrema positions (o symbols). . . . . . .

8 Decision rule for merging a pair of neighboring elementary ridge segments: (a) validated merging, (b) rejected merging. The pair of tested segments is displayed by a solid line, while neighboring elementary valley segments are depicted by dashed lines. . . .

9 Illustration of the computation of the envelope growth model from a set of examples of plaice (Pleuronectes Platessa) growth patterns for the year class $k=4$ : (a) envelope (black dashed plots) computed from the growth patterns, (b) envelope (black dashed plots) computed from their first-order derivative. . . . . . . . . . . . . .

10 Illustration of the evaluation of the relevance of growth pattern examples given a variability range set by the envelope $g_{\min }^{k}, g_{\max }^{k}$ for the age class $k=4$ : (a) relevant growth pattern, (b) irrelevant growth pattern. . . . . . . . . . . . . . .

11 Constraint on the spatial arrangement of the selected rings curve: relevant (a) vs. irrelevant (b) configurations. Pair of successive ring curves are required to depict valley and ridge curves since the interpretation of growth rings has to refer to an alternation of opaque and translucent rings. $\ldots \ldots \ldots \ldots \ldots \ldots$ 
12 Extractions of meaningful ring structures within images of biological hard tissues depicting growth ring structures. The first row displays an application to a fish otolith $(B a r=1 \mathrm{~mm})$, the second to the shell of a bivalve seashell $(B a r=0.5 \mathrm{~cm})$ and the third one to the section of a tree trunk $(\mathrm{Bar}=10 \mathrm{~cm})$. The first column provides the original images, the second one the extracted elementary ridge and valley segments, and the third one the detected ring curves resulting from the grouping of the elementary

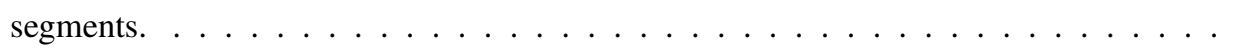

13 Extractions of meaningful ring structures within images of otolith images depicting ontogenetic changes in the shape of the rings: the first row displays an application to a pollock otolith, and the second one to a cod otolith. The first column displays the original images, the second one the extracted elementary ridge and valley segments, and the third one the detected ring curves resulting from the grouping of the elementary segments. $\mathrm{Bar}=1 \mathrm{~mm} . \ldots \ldots \ldots \ldots \ldots \ldots \ldots$

14 Extraction of closed ring curves using a global template-based scheme (Troadec et al. 2000) for the two images processed using our semi-local template-based approach in Fig.13: (a) pollock otolith, (b) cod otolith. Bar $=1 \mathrm{~mm} \ldots \ldots \ldots \ldots \ldots$

15 Interpretation of images of plaice (Pleuronectes Platessa) otoliths for three examples: the first row depicts the otolith images and the associated expert interpretations, the second one the extracted ridge curves, the third one the curve subsets selected for age and growth estimation and the fourth one the estimated growth patterns (solid line) compared to the ground truth ones (dashed line). For each example, the ground truth is provided by the interpretation of an expert in plaice (Pleuronectes Platessa) otolith reading. $\mathrm{Bar}=1 \mathrm{~mm} . \ldots \ldots \ldots \ldots \ldots \ldots$ 
16 Age estimation errors for the test set of 200 images of plaice (Pleuronectes Platessa) otoliths: (a) age composition of the processed otolith dataset, (b) rates of the age estimation error for age class 1 to 6 , (c) mean rate of the age estimation error. . . . . . . . 46

17 Influence of errors in pointing the actual growth center for the extraction of ridge and valley curves (we only display the extracted ridge curves): on the left, the actual position of the otolith center is used; on the right, the center is translated of 30 pixels to the left and 20 to the top w.r.t. the actual growth center. $B a r=1 \mathrm{~mm} . \ldots \ldots \ldots$

18 Examples of two disagreements in age reading between the expert and the proposed approach. The first row displays the processed otolith image with the markers (०) associated to the interpretation of the expert. The second row depicts the otolith interpretation resulting from the Bayesian selection of the extracted ridge and valley curves (we only display the selected ridge curves) $\ldots \ldots \ldots \ldots \ldots \ldots$ 
Fig. 1.
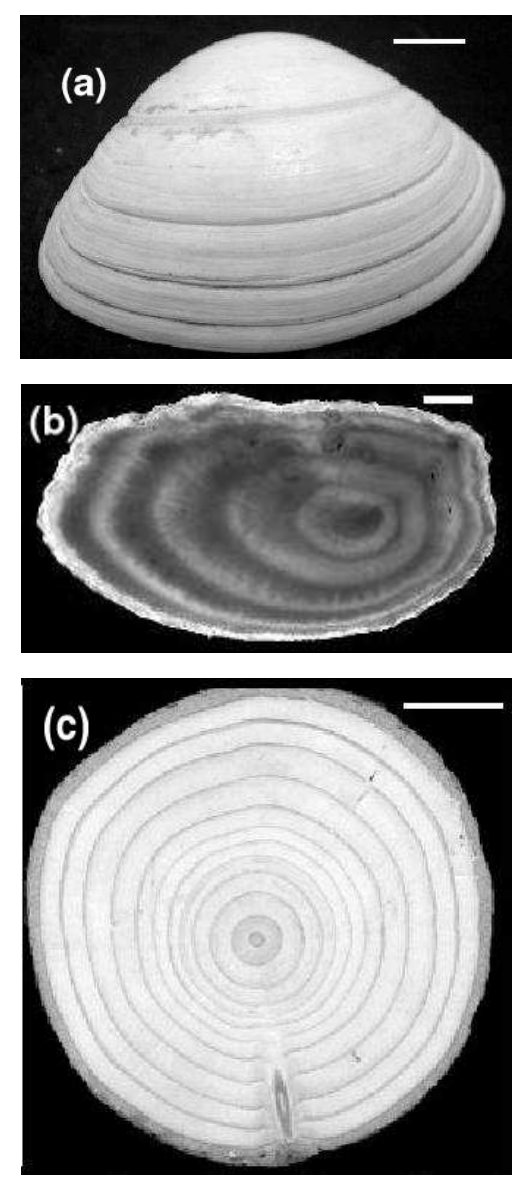
Fig. 2.

(a)

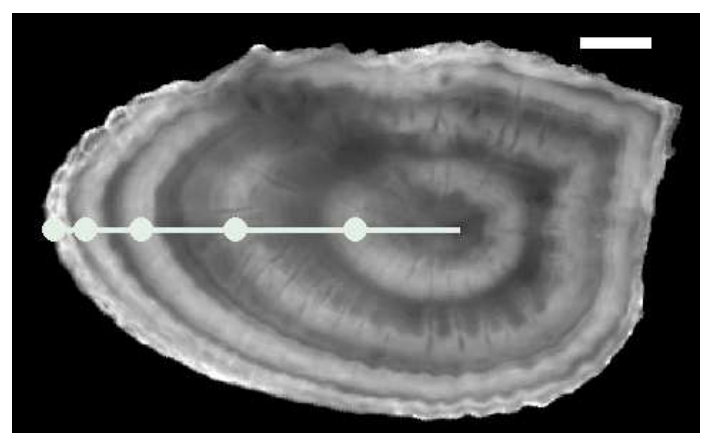

(b)

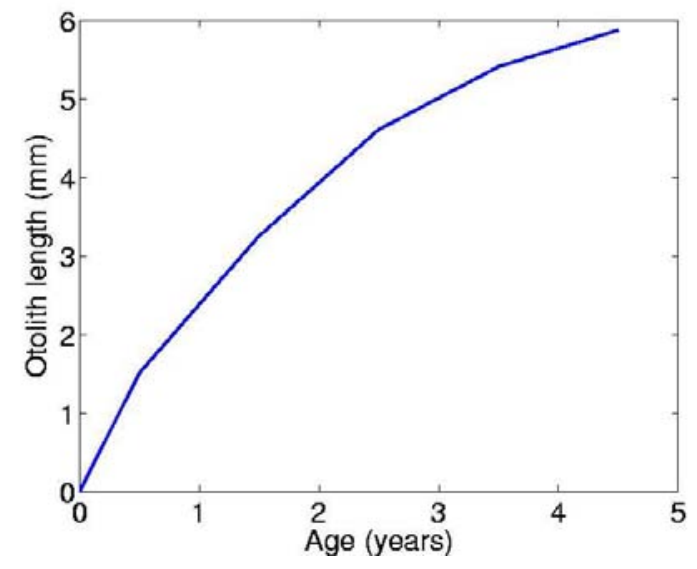


Fig. 3.

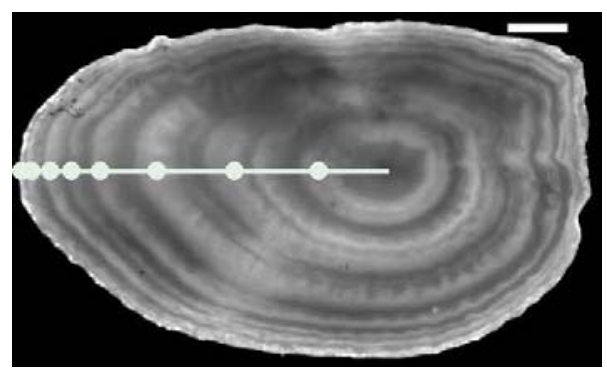

(C)2005 NRC Canada 
Fig. 4.

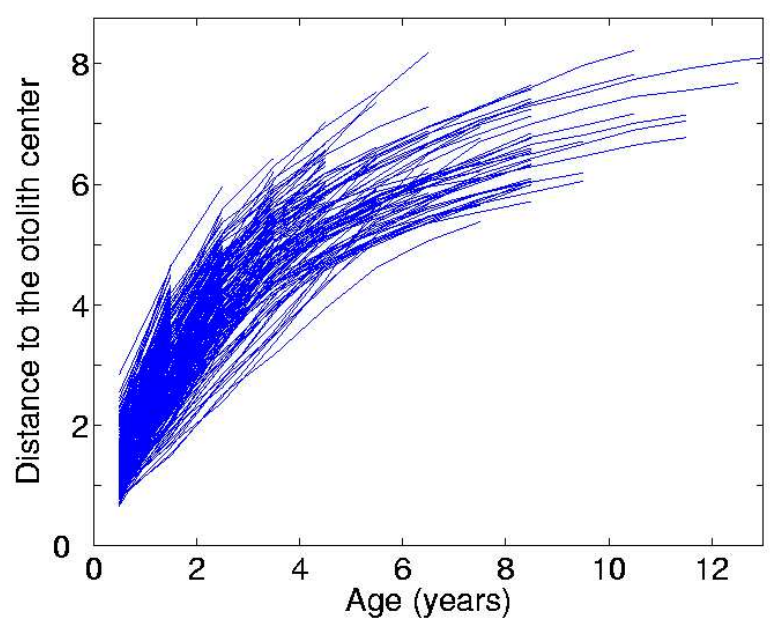

(C) 2005 NRC Canada 
Fig. 5.
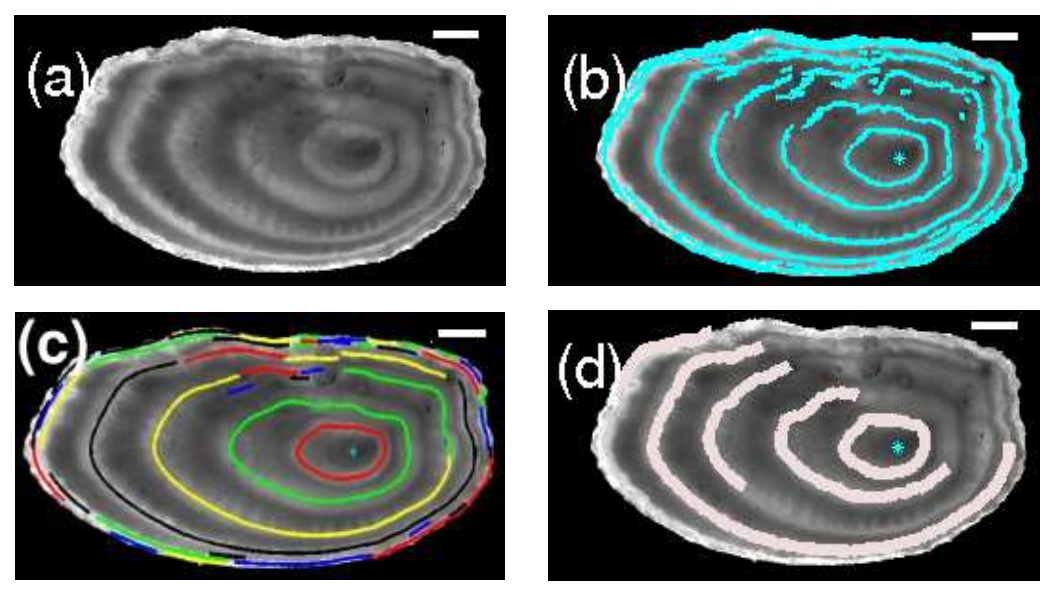

(C) 2005 NRC Canada 
Fig. 6.

(a) first principal axis

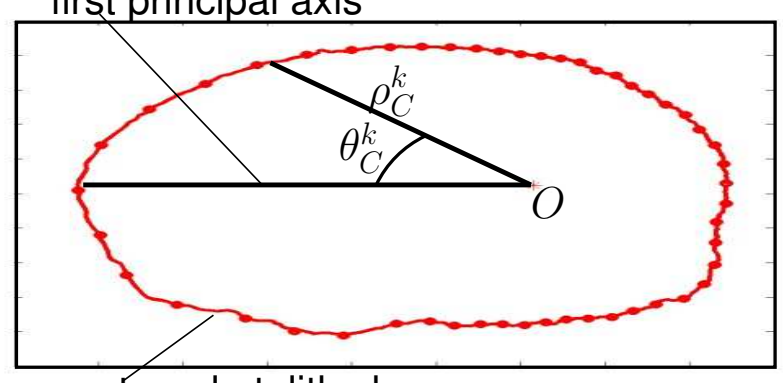

external otolith shape

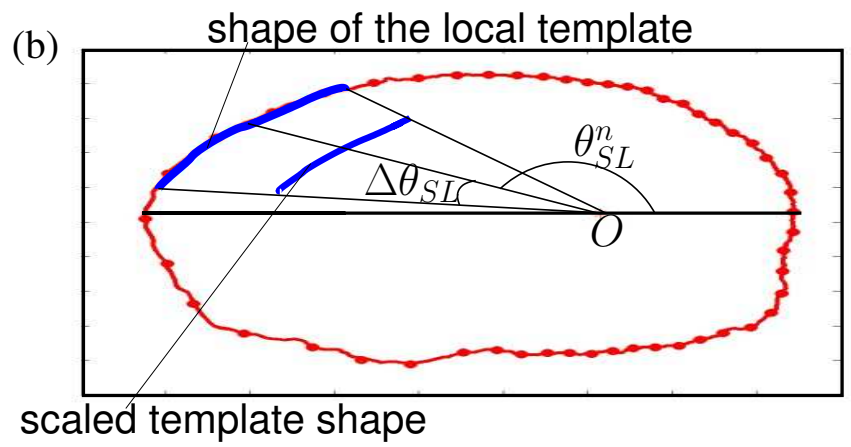


Fig. 7.

(a)

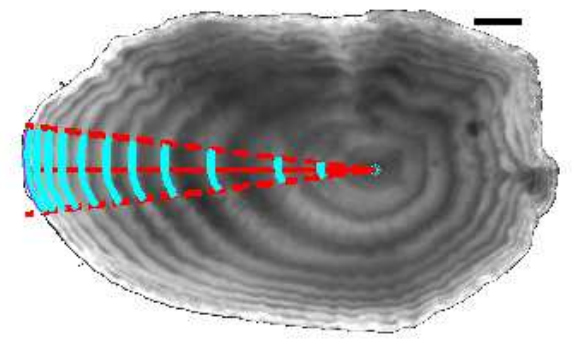

(b)

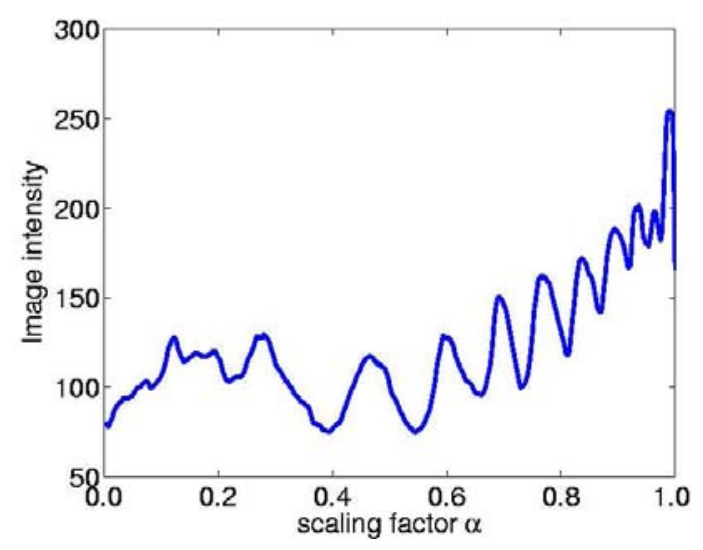

(c)

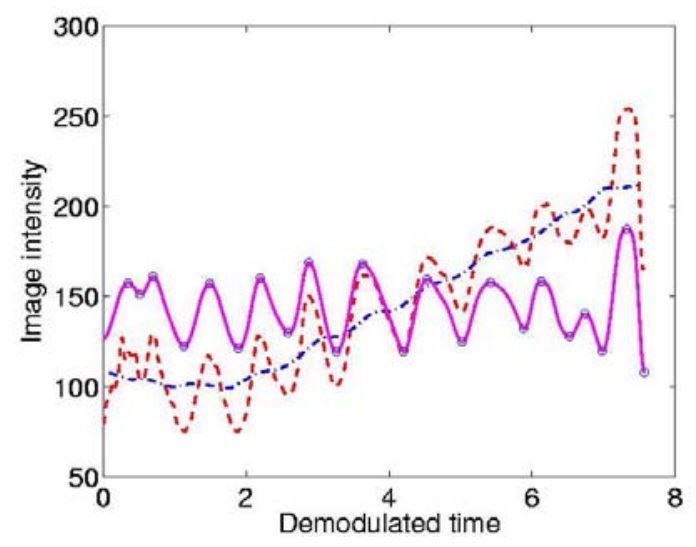


Fig. 8.

(a)

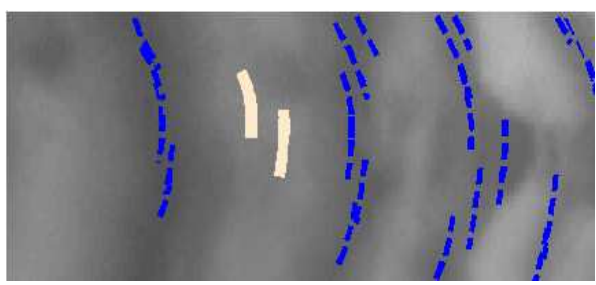

(b)

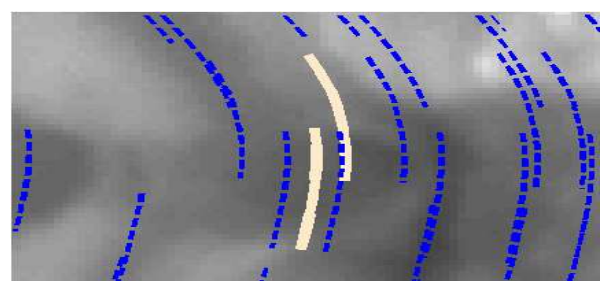

Fig. 9.

(a)

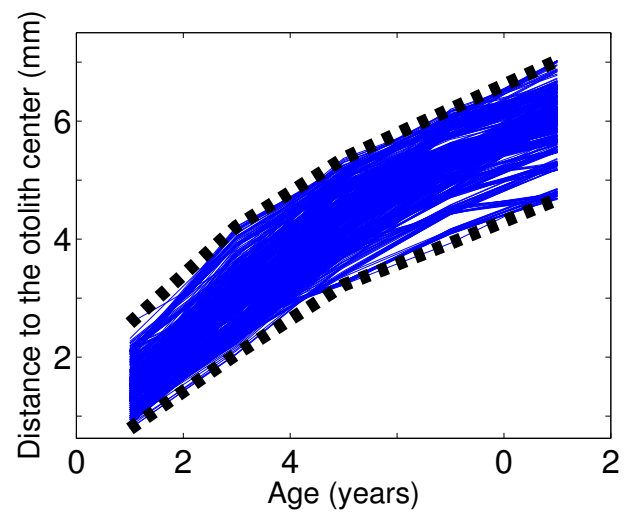

(b)

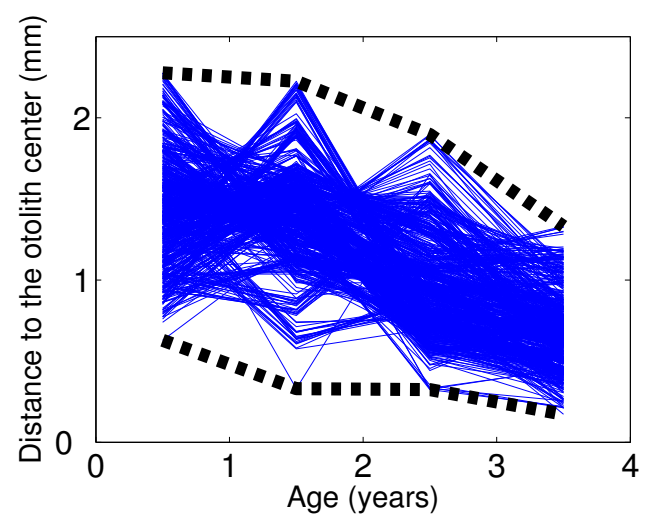


Fig. 10.

(a)

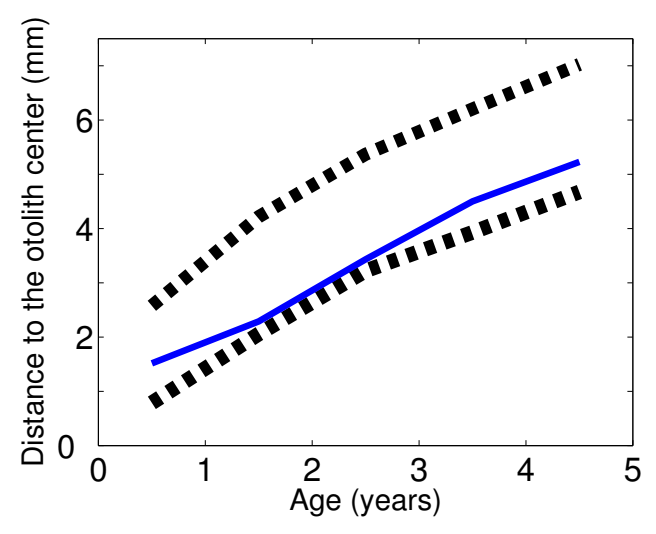

Fig. 11.

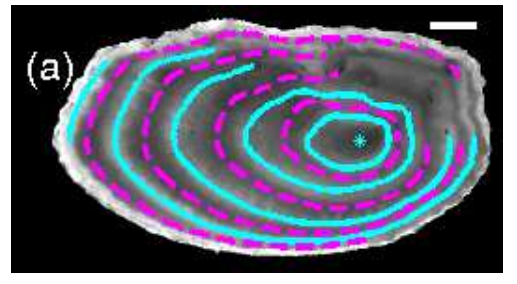

(b)
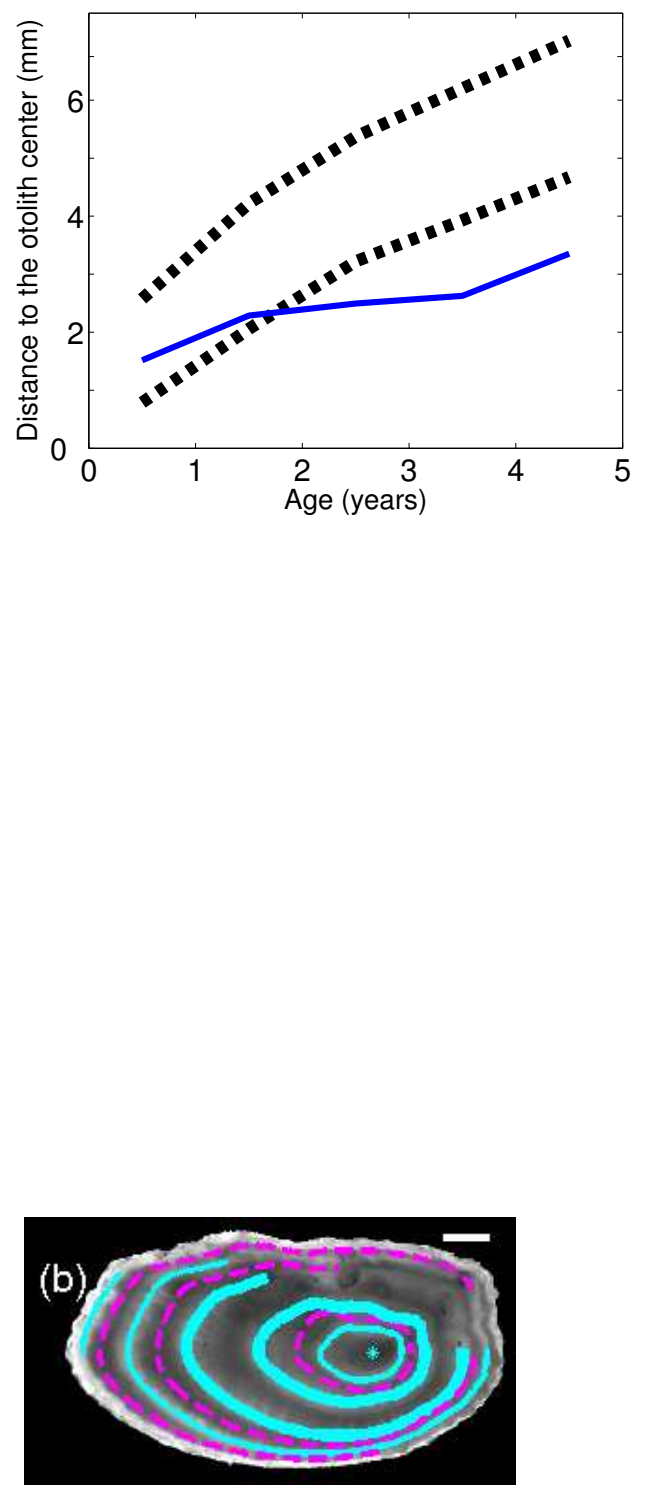
Fig. 12.
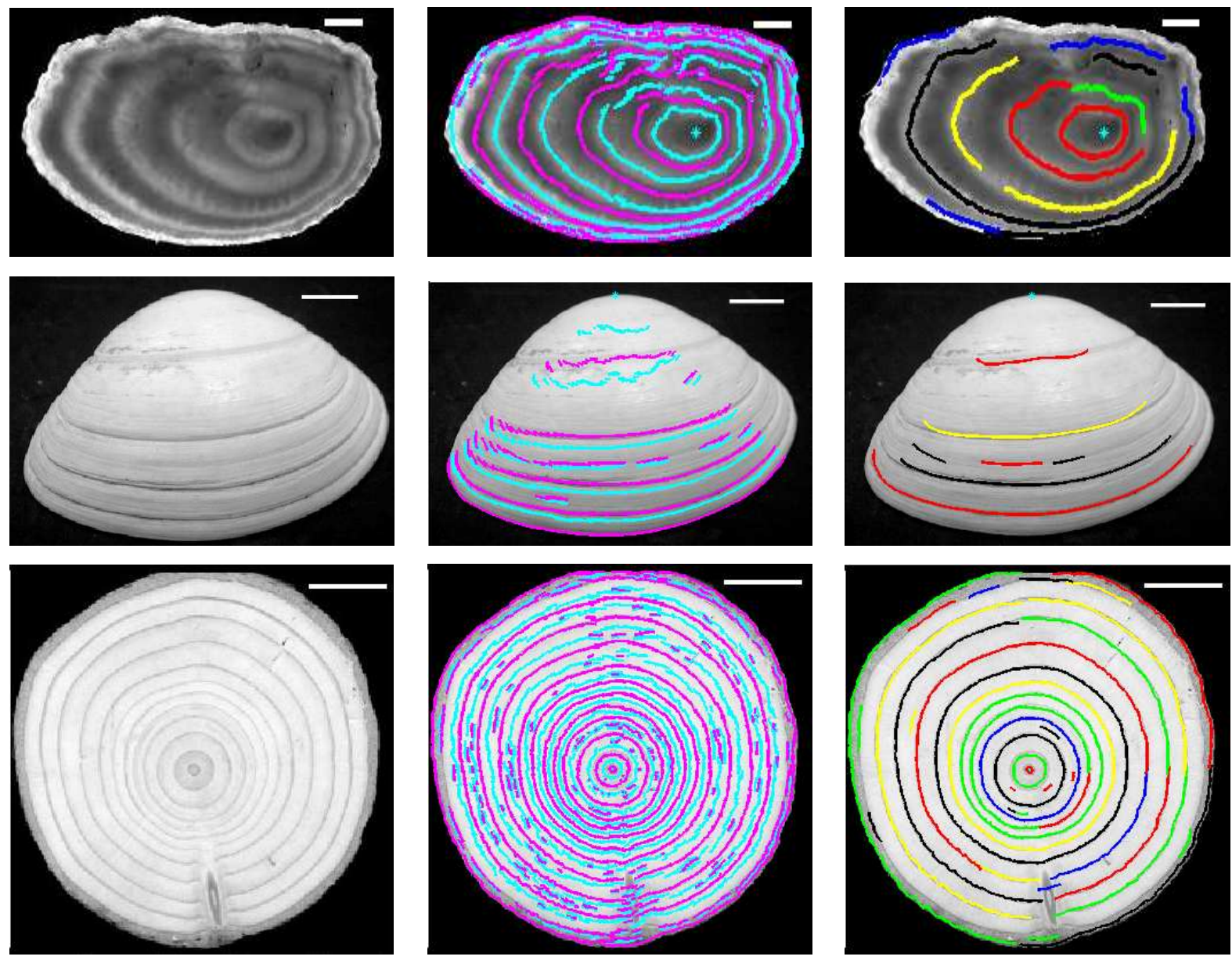

(c)2005 NRC Canada 
Fig. 13.
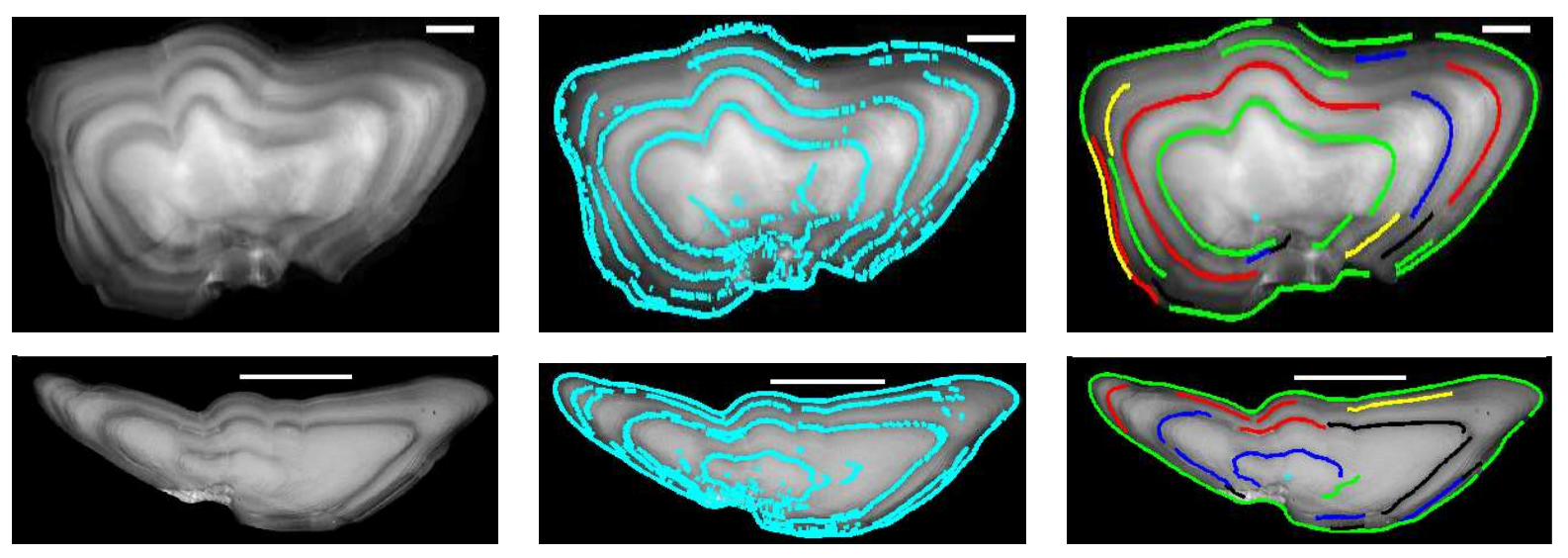

Fig. 14.

(a)

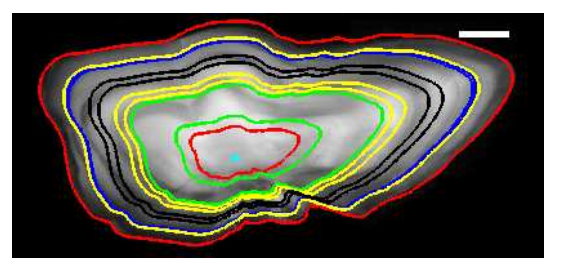

(b)

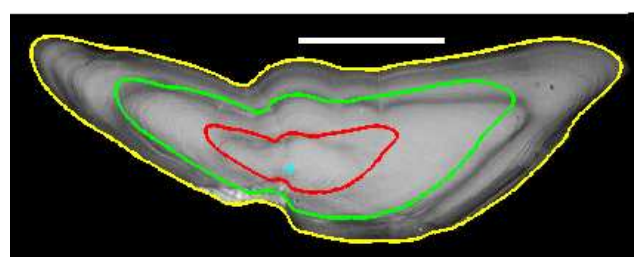


Fig. 15.
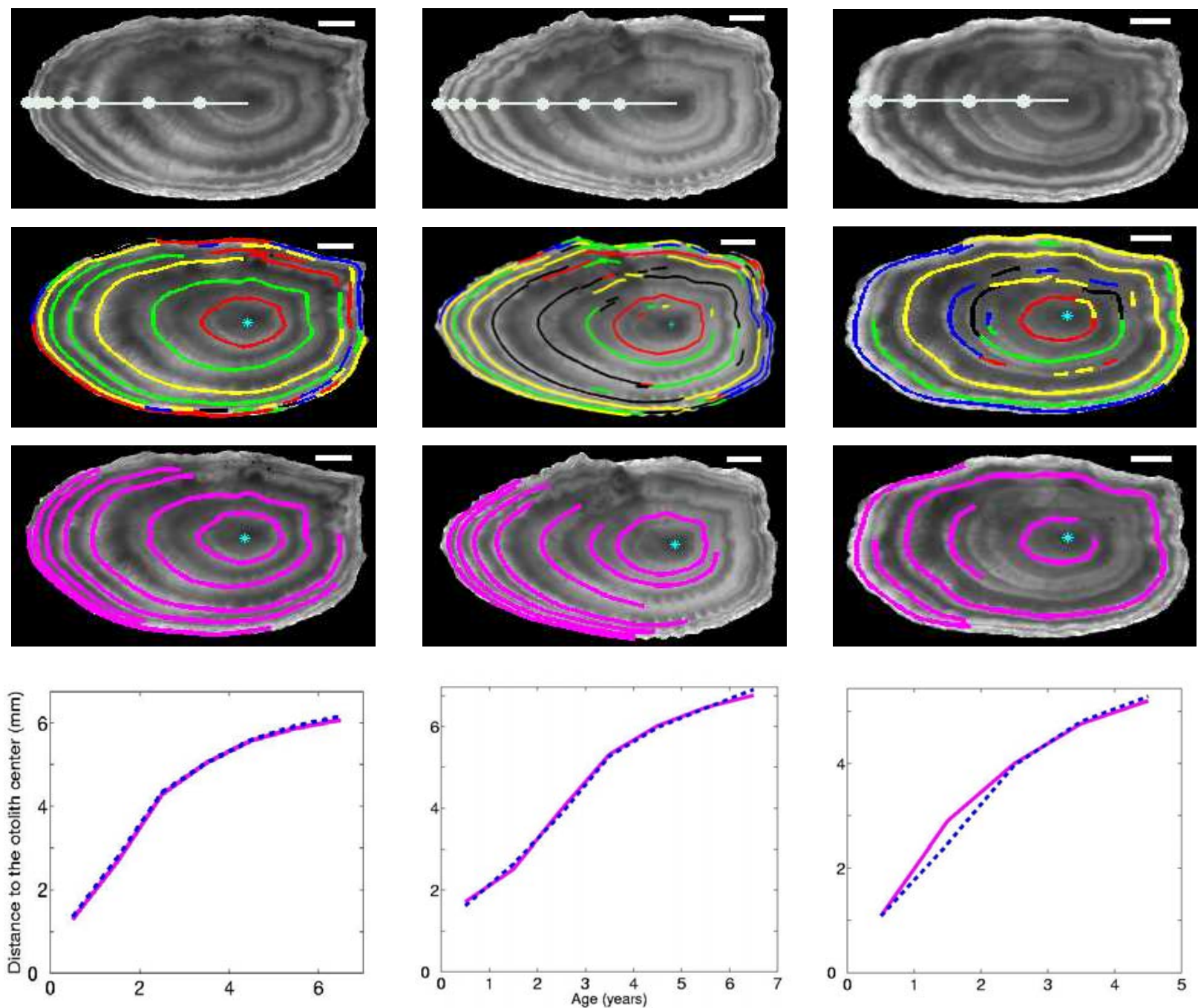

(c) 2005 NRC Canada 
Fig. 16.

(a)

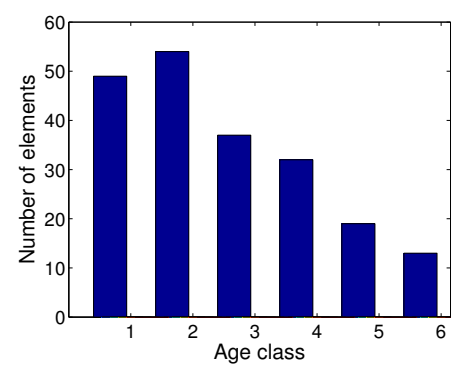

(b)

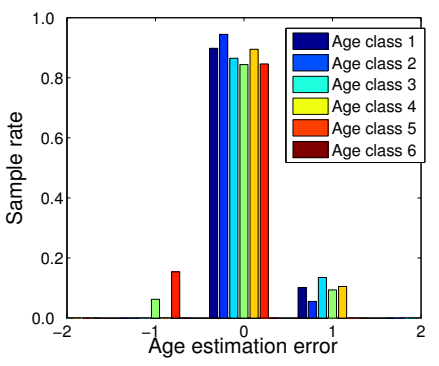

(c)

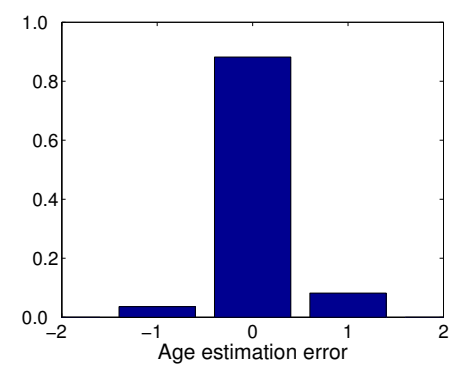


Fig. 17.
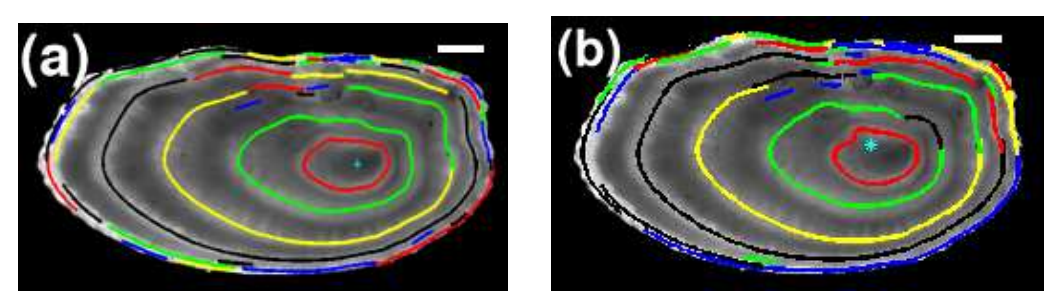

Fig. 18.
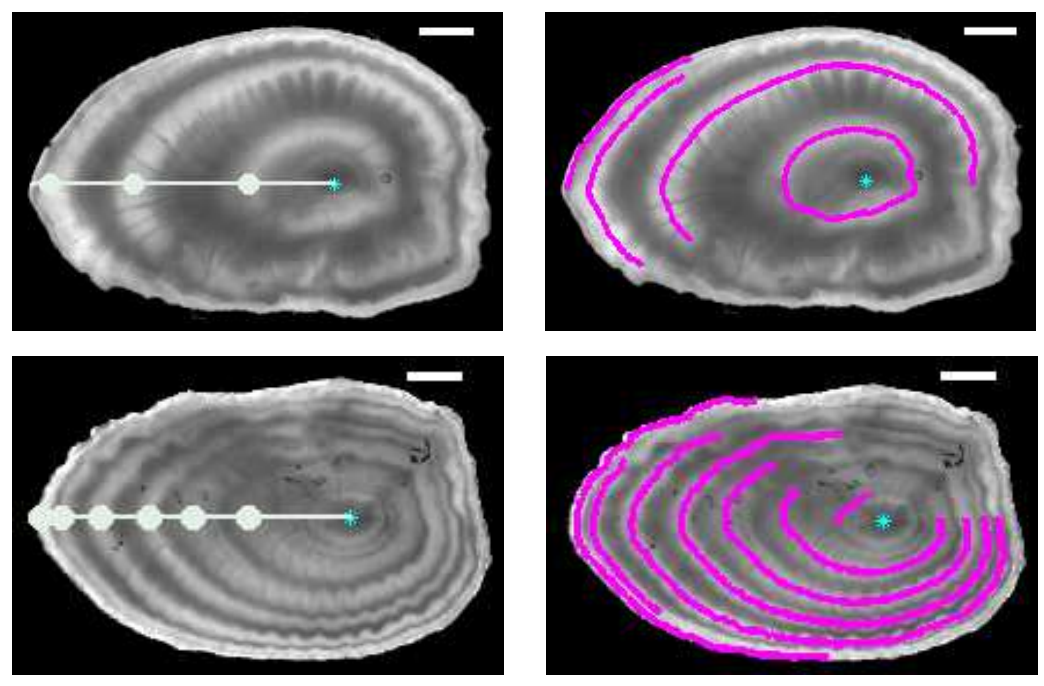

(C)2005 NRC Canada 